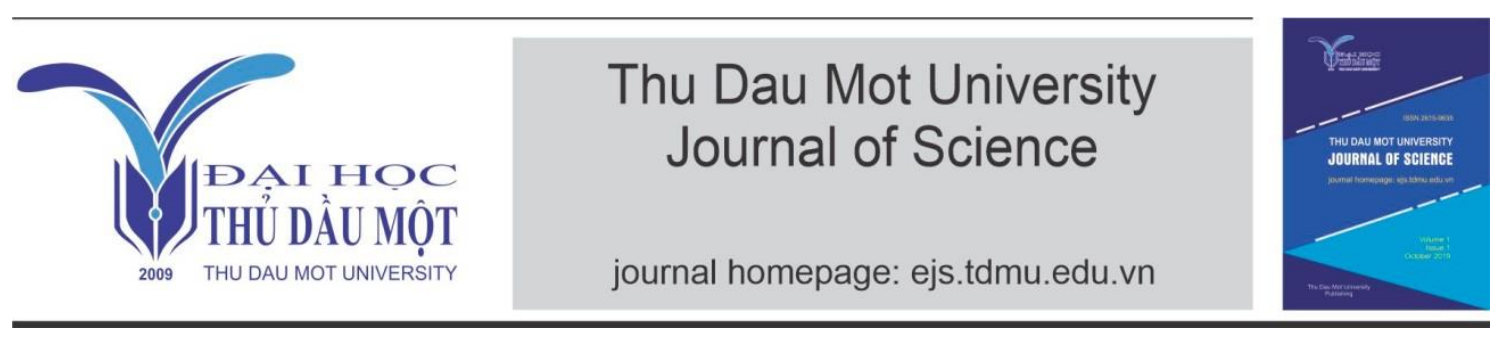

\title{
An Overview of Emerging Technologies for 5G: Full-Duplex Relaying Cognitive Radio Networks, Device-to-Device Communications and Cell-free Massive MIMO
}

by Toan Xuan Doan, Thanh Quoc Trinh (Thu Dau Mot University)

Article Info: Received 16 Aug 2020, Accepted 24 Oct 2020, Available online 15 Dec, 2020

Corresponding author: toandx@tdmu.edu.vn

https://doi.org/10.37550/tdmu.EJS/2020.04.080

\begin{abstract}
The fifth generation $(5 G)$ cellular network has been commercialized recently to fulfill the new demands such as very high data exchange rate, extra low latency and high reliability. Many new technologies have been introduced and exploited since the early of the 2010s. Among these emerging technologies, full-duplex relaying cognitive radio networks, device-to-device communications and cell-free massive multiple-input and multiple-output have been considered as promising technologies/systems for $5 G$ and beyond. This work provides a comprehensive study on the concepts, advantages and challenges of the above-mentioned technologies. In addition, we also introduce four new research directions which are challenges of $5 G$ and beyond.
\end{abstract}

Keywords: Cell-free massive MIMO, full-duplex relaying cognitive radio networks, device-to-device communications

\section{Introduction}

Since the last two decades, we have witnessed an impressive and unpredictable growth of wireless cellular networks. In 1982, a mobile communication system was first introduced, and it was considered as a luxuriously fashionable service. This version, 
namely first generation $(1 \mathrm{G})$, was based on analogue technology, and was only able to provide voice service. In the 1990s, the second generation of cellular networks, namely $2 \mathrm{G}$, was launched. Owning to the advantages of digital technique, $2 \mathrm{G}$ could support both voice and text services. Then, $2 \mathrm{G}$ quickly became popular and appeared in daily lives, and the demand for accessing the internet was growing. This was the motivation of the third generation's emergence in 2001 (during the developing of 3G, two enhanced versions of $2 \mathrm{G}$ also were introduced [1]). 3G opened a new horizon for data transmission with package switching technology. After that an updated version of $3 \mathrm{G}$, namely high-speed downlink packet access, which was able to support a download data rate up to $14 \mathrm{Mbit} / \mathrm{s}$, was introduced. In 2012, 4G was standardized. Long Term Evolution Advanced, a version of $4 \mathrm{G}$, can provide download and upload data rate up to $1 \mathrm{Gbit} / \mathrm{s}$ and $500 \mathrm{Mbit} / \mathrm{s}$, respectively. After appearing several years, 4G systems became unable to fulfill the high demand on capacity, reliability and low latency, particularly in the emergence of many new applications and related technologies such as Internet-of-things, autonomous driving and video streaming. Thus, the fifth generation (5G) mobile networks, which is expected to appear in 2020, have been under intensive research and development [2],[3].

Since 2012, many infrastructure vendors (such as Ericsson, Huawei and Samsung) and governments have considered to establish a definition and features for 5G. Although there are various versions of 5G's vision, the key points are summarized as follows [2]:

- High data rates: $10 \mathrm{Gbps}$ in the real networks.

- Low latency: less than 5 milliseconds round strip delay.

- Increasing bandwidth: bandwidth per unit area is expected to increase 1000 times.

- Massive connectivity.

- Ultra-high reliability.

- Availability: $100 \%$ coverage.

- Energy efficiency: reducing $90 \%$ of network energy consumption.

- Low power: prolong-life of battery up to ten years.

\section{Advantages and challenges}

Based on the 5G's vision, many studies have been done and many solutions related to the radio access network have been proposed, such as cell-free (CF) massive multipleinput multiple-output (MIMO), cognitive radio network (CRNs), full-duplex relaying system (FDR), device-to-device (D2D) communication and energy harvesting communication (EHC). These emerging techniques are considered as key components of $5 \mathrm{G}$ and beyond architecture. Table 1 shows a summary of some key advantages and 
challenges of these technologies [4]-[11]. It shows that interference is still a key challenge for the future wireless communication systems.

- CF massive MIMO: Deploying unpaired wise orthogonal pilot signals causes pilot contamination effect which significantly reduces the system performance even when the number of access points is large. In addition, multi-user interference is also a challenge.

- D2D communications: For underlay in-band D2D communications, D2D users and cellular users simultaneously operate in the same spectrum, leading to interference between their communications.

- FDR: It is difficult to perfectly cancel self-interference in the real network. Self-interference still exists.

- CRNs: Secondary users share spectrum with primary users leading to increase spectrum efficiency along with causing interference.

$T A B L E$ 1. Key features of the emerging techniques

\begin{tabular}{ccll}
\hline No & Techs & \multicolumn{1}{c}{ Advantages } & \multicolumn{1}{c}{ Challenges } \\
\hline 1 & CRNs & High spectral efficiency. & $\begin{array}{l}\text { Interference between primary and Secondary } \\
\text { networks, spectrum sensing mechanisms. }\end{array}$ \\
\hline 2 & D2D & $\begin{array}{l}\text { High spectral efficiency, low } \\
\text { latency. }\end{array}$ & Interference, D2D user discovery. \\
\hline 3 & FDR & High coverage, data rate & Self-interference. \\
\hline 4 & $\begin{array}{c}\text { CF massive } \\
\text { MIMO }\end{array}$ & $\begin{array}{l}\text { Uniformly high spectral efficiency } \\
\text { and energy efficiency. }\end{array}$ & $\begin{array}{l}\text { Pilot contamination, backhaul links } \\
\text { requirements. }\end{array}$ \\
\hline
\end{tabular}

\section{Relevant studies}

In the literature, CRNs, FDR, D2D and CF massive MIMO have been intensively studied.

- The authors in [4] and [5] gave a brief overview on CRNs, then discussed the technologies that dealt with four main challenges of CRNs i.e. spectrum sensing, spectrum decision, spectrum sharing and spectrum mobility. Particularly, the architecture and applications of CRNs in detail were presented in [5], making CRNs close to the reality.

- FDR, a novel design for precoder and decoder, was introduced in [12] to overcome self-interference at a FD relay. The results showed that by applying the proposed precoder/decoder, the system performance in terms of achievable rate and bit error rate was enhanced significantly in comparison with half- 
duplex relays and conventional FD relays. Another work, [13], also focused on interference reduction method using a simple adaptive feedback canceller for amplify-and-forward relays based on second-order statistics.

- Along with many works concentrating on improving spectrum efficiency, fairness and reliability of a D2D system, there are other works to address technical challenges such as resource allocation, D2D users discovery and interference (underlay in-band D2D) [8].

- CF massive MIMO has attracted a great attention recently [9]-[11], [14], [15]. $\mathrm{CF}$ is considered as a next research direction after massive MIMO [11].

From the aforementioned discussion, an overview of these technologies in terms of the concepts, advantages and disadvantages is discussed in the paper. The paper is divided into five sections. The introduction is presented in the first section, the conclusion is in Section 5. The main discussions are presented in three technical sections including CF massive MIMO, FDRs in CRNs and D2D communications.

For each technical section, the content consists of introduction, relative works, advantages and disadvantages of the corresponding technique.

\section{Cell-free massive MIMO}

To increase the network connectivity and improve the spectral efficiency, cells are divided into smaller cells. However, splitting cells creates a new issue that is inter-cell interference. This negative effect becomes more severe when the cell radius is small. Consequently, the capacity is limited. In addition to that, inter-cell interference also makes cell edge users' performance become worse (see Fig. 1).

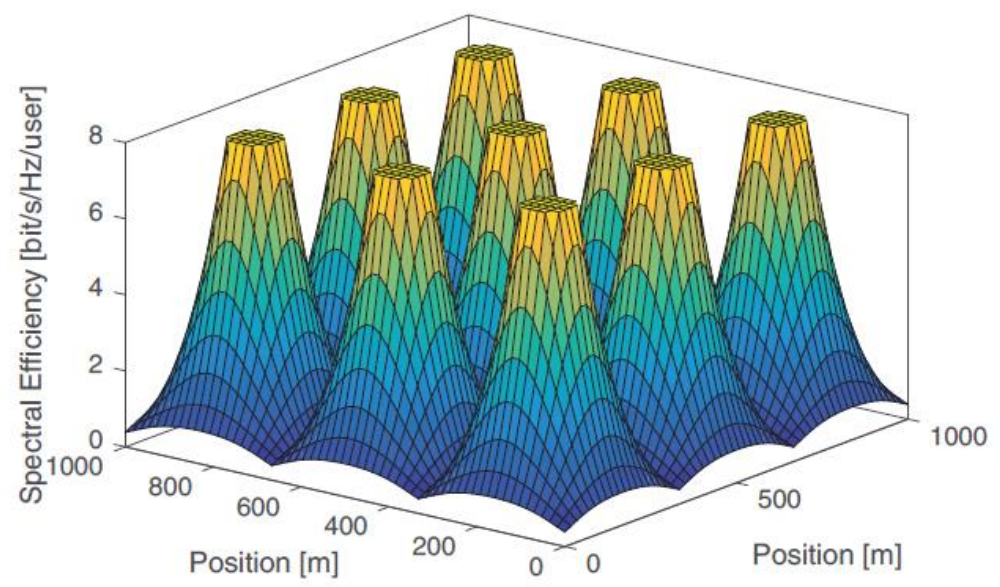

Figure 1. The spectral efficiency in small cell systems [16]. 
To overcome this restriction, a co-processing each signal at multiple base stations (BSs) technique has been considered. The principle of this technique is to turn inter-cell interference to be a desired signal through using neighbor BSs to co-serve a user or many users [16]. Such system has been introduced under different names, such as coordinated multipoint with joint transmission (COMP-JT) [17] and distributed wireless communication system [18]. Although many theoretical works proved that this approach could provide great advantages, it has not been applied in practice up to now.

Recently, a new concept called CF massive MIMO has been proposed in [9]. This concept originates from massive MIMO, but massive antennas are spread out over a wide area instead of being located in a compact area. It has attracted a great attention and many studies have been performed. The work in [11] proposed the expressions for achievable uplink and downlink rate. More interestingly, the authors compared between $\mathrm{CF}$ system and small cell in terms of performance of downlink/uplink network throughput. The results showed that CF system provided better throughput performance than small cell did (see Fig. 2). In addition to that, the cell-edge users in CF system also do not suffer poor spectral efficiency, (see Fig. 3). Furthermore, some other works focused on other aspects of $\mathrm{CF}$ system, such as proposing a pilot assignment protocol for CF system [11] or dealing with limited back-haul in CF system [15].

\subsection{Cell-free massive MIMO system model}

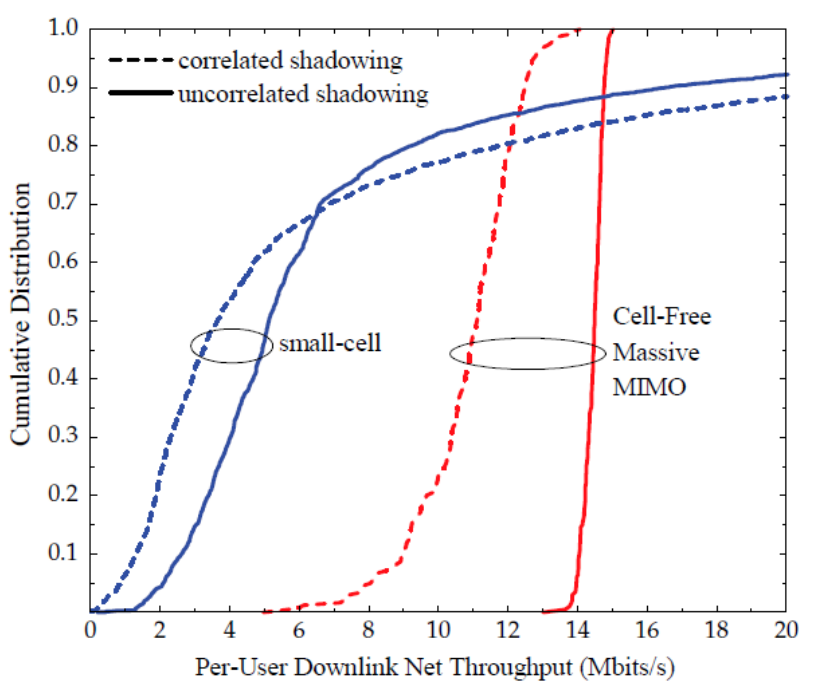

Figure 2. The cumulative distribution of per-user downlink net throughput [11].

As discussed in the previous part, CF massive MIMO system is the distributed massive MIMO system, where massive antennas spread over a large area, named as access point (AP), serving a much smaller number of users in the same time-frequency resources. A central processing unit (CPU) connecting to all APs via a back-haul links is deployed 
for processing most of important tasks, including decoding uplink data, coding downlink data, and power control (see Fig. 4).

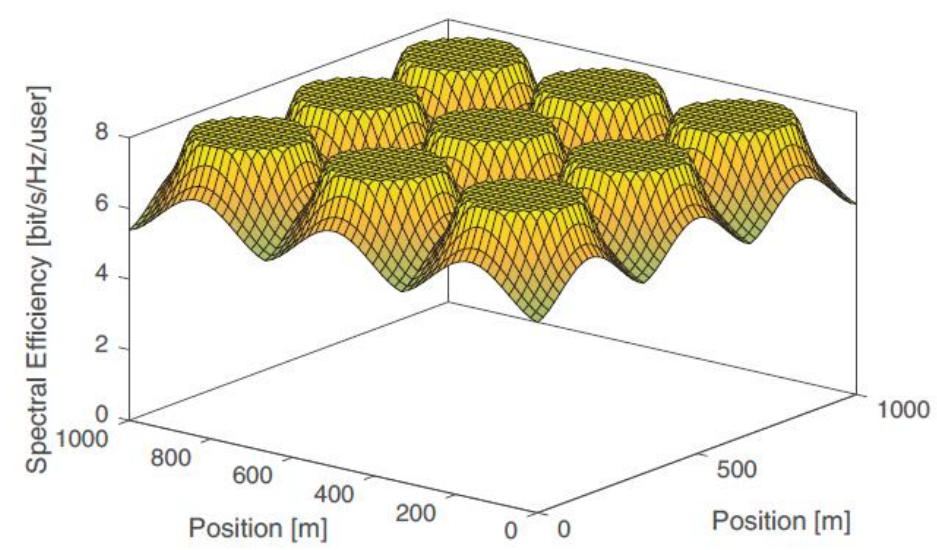

Figure 3. The spectral efficiency at the cell edges in CF massive MIMO [16].

Similar to conventional massive MIMO, time division duplex (TDD) protocol is applied in CF. In the first phase, namely pilot uplink training, the users send pilot signal to APs, and APs estimate the channels state information (CSI). This estimated information is useful for decoding and beamforming. In the second phase, namely downlink payload transmission, APs send data to the users. In the third phase, namely downlink pilot training, APs broadcast a pilot signal to users in order to help the users obtain the CSI. Interestingly, when the number of APs is sufficiently large, the channel from APs to user $\mathrm{k}$ becomes hardening. In other words, the channel approximates to its expectation value [11]. Thus, the third phase does not require in this case [11]. The last phase, named uplink data transmission, users send data to APs. The received data at APs is pre-processed before being forwarded them to CPU to detect the desired information. Generally, the equations of uplink and downlink data are given as follows:

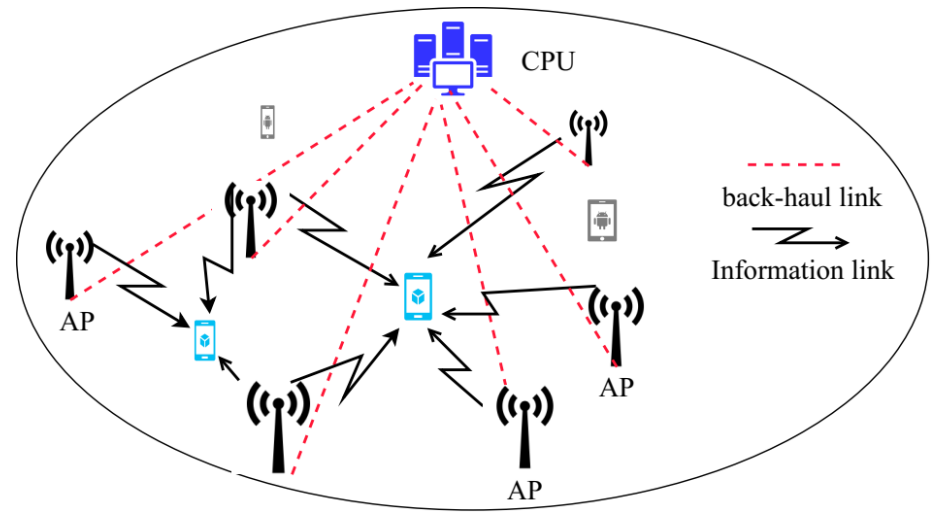

Figure 4. CF massive MIMO system model. 
Downlink transmission: The received signal at user $k$ is a sum of signals from all APs and it is given by

$$
r_{k}^{\mathrm{dl}}=\sum_{m \in \mathrm{A}} \sum_{k^{\prime} \in \mathrm{U}} \sqrt{\rho_{m}^{\mathrm{AP}} \eta_{m k}^{\mathrm{dl}}} g_{m k} z_{m k^{\prime}} q_{k^{\prime}}+w_{k}
$$

where $A$ and $U$ are the set of APs and users, respectively, $\rho_{m}^{\mathrm{AP}}$ is the transmit power of $m^{\text {th }} \mathrm{AP}, \eta_{m k}^{\mathrm{dl}}$ is the power control coefficient, $g_{m k}$ is the channel coefficient between $m^{\text {th }}$ AP and user $k, z_{m k^{\prime}}$ is the beamforming component, $q_{k^{\prime}}$ is the symbol needed to send to user $k, w_{k}$ is the AWGN.

Uplink transmission: The received signal of user $k$ at CPU is expressed as

$$
r_{k}^{\mathrm{ul}}=\sum_{m \in \mathrm{A}} \sum_{k^{\prime} \in \mathrm{U}} \sqrt{\boldsymbol{\rho}_{m}^{\mathrm{u}} \eta_{m k}^{\mathrm{ul}}} g_{m k} \delta_{k^{\prime}}+\sum_{m \in \mathrm{A}} w_{k}
$$

where $\rho_{m}^{\mathrm{u}}$ is the transmit power of user $k, \eta_{m k}^{\mathrm{ul}}$ is the weight coefficient of $\mathrm{AP} m, \delta_{k^{\prime}}$ is the transmit symbol of user $k^{\prime}$.

\subsection{Discussion on CSI Exchange in Cell-free massive MIMO}

Conjugate beamforming is suggested so that APs can perform beamforming locally. In addition, power control coefficient depends on large-scale fading which changes slowly. Thus, it does not need to exchange instantaneous CSI between CPU and APs [11].

\subsection{Challenges in Cell-free massive MIMO}

CF massive MIMO inherits advantages from conventional massive MIMO and from coprocessing each signal at multiple APs/BSs technique. It also owns challenges of these systems, including requirement of sufficiently accurate CSI, time synchronization and high quality of back-haul link connecting APs to CPU [11].

\section{Full-duplex relaying cognitive radio networks}

CRNs, which was first introduced by J. Mitola [19], is a solution for improving the spectral efficiency [4], [5], [20]. The key principle of this concept is a secondary user (SU) (unlicensed user) shares the spectrum of a primary user (PU) (licensed user) (see Fig. 5). In other words, SU is allowed to access the licensed spectrum without causing harmful influence on the PU communication. To efficiently manage spectral resource, many approaches have been proposed, such as underlay, overlay and interweave policies [21]. 


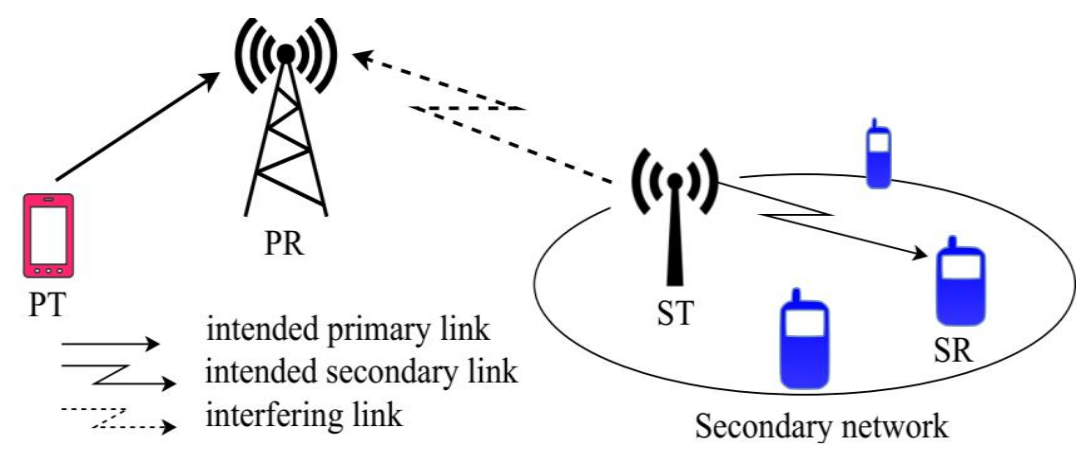

Figure 5. CRNs system with underlay paradigm

\subsection{Interweave paradigm}

The motivation behind this scheme is that there exists some temporary spectrum unutilized, called as spectrum holes so that by utilization these holes the spectral efficiency is enhanced. Because the availability of spectrum holes changes over time, the SUs need to have some protocols to detect and access to the spectrum holes [22], [23].

\subsection{Underlay paradigm}

To ensure the PU still meets its quality of service (QoS), the SUs have to know the CSI of the link to primary receivers, to control the transmit power so that the peak interference caused by the SU on PUs drops below a given threshold (interference constraint) [21]. Due to the limitation in transmit power, underlay scheme suits for short range transmission at the secondary networks, the authors of [24] proved that SU might obtain very high rate with low transmit power.

\subsection{Overlay paradigm}

This policy also allows SUs and PUs to transmit signal simultaneously at the same spectrum. As SUs can transmit signal with any power, it is needed to have some techniques to partly or completely cancel interference. Thus, SUs need to obtain more information about PUs, such as CSI, codebooks and message of primary transmitters [21].

Relaying system is introduced in order to extend the coverage of a cellular network without increasing transmit power at transmitters. Particularly, this system can help cell-edge users improve their performance, which is one of the challenges in cellular networks [25]-[27]. The operation principle of relays is to receive signal from a transmitter and then forward the received signal to desired destination.

Relays operate under two modes including half-duplex (HD) and full-duplex (FD). For HD, relays receive signal and forward the received signal in two different phases/timeplots (or in the same time-slot but different frequencies). For FD, relays transmit and receive signal at the same time and frequency, i.e. when the relay forwards signal, 
which was received in the previous phase, to the destination, it also receives signal from the transmitter (as Fig. 6 and Fig. 7). As a consequence, FD can offer a better spectrum efficiency at the price of experiencing self-interference (SI) at the relay, i.e. the signal in the receiving side is interfered by transmitted signal in the transmission side. For that, an interference cancellation approach is needed for FD [12], [13], [28]. With a sufficiently efficient SI cancellation, the data rate can be double [7].

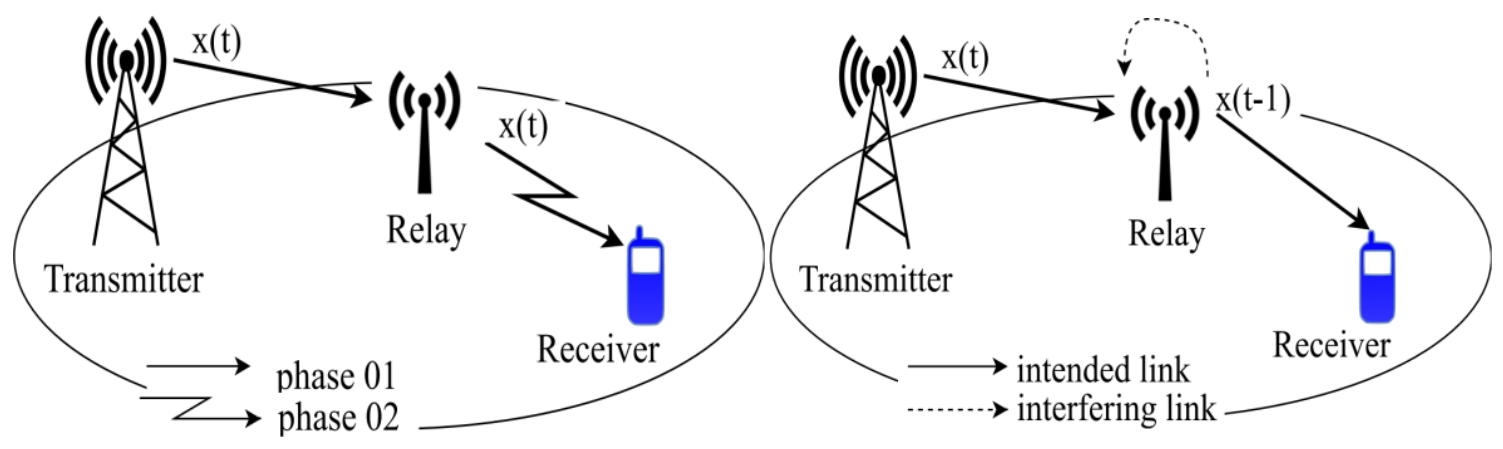

Figure 6. HD relaying system

Figure 7. FD relaying system

Fig. 6 demonstrates a HD relaying system, where a transmit signal $x(t)$ is transmitted to relay in phase 01 , and then the relay forwards $x(t)$ to a receiver in phase 02. Fig. 7 demonstrates a FD relaying system, where relay transmits the signal $x(t-1)$, which is received in the previous phase, to the receiver. At the same time, relay also receives signal $x(t)$ from the transmitter. Due to operating in FD mode, the receiving side of the relay is interfered by the transmitting side.

At the relay, after receiving signal, the relay forwards it to destination based on two common protocols: amplify-and-forward (AF) and decode-and-forward (DF). The advantages of AF protocol is that this process is simpler than DF. However, interference and noise of the transmitter-relay hop are also amplified, which results in decreasing SINR value at the destination.

The instantaneous end-to-end SNRs of a two-hop AF relaying system, denoted by $\mathrm{g}_{e 2 e}^{A F}$ and of a two-hop DF relaying system, $\mathrm{g}_{e 2 e}^{D F}$ are given by [29], [30],

$$
\mathrm{g}_{e 2 e}^{A F}=\frac{\mathrm{g}_{s r} \mathrm{~g}_{r d}}{1+\mathrm{g}_{s r}+\mathrm{g}_{r d}}, \mathrm{~g}_{e 2 e}^{D F}=\min \left\{\mathrm{g}_{s r}, \mathrm{~g}_{r d}\right\}
$$

where $\mathrm{g}_{s r}$ and $\mathrm{g}_{r d}$ are the instantaneous SNRs of the first hop from source (transmitter) to relay and the second hop from relay to destination, respectively.

Interestingly, a new concept of relay station has been proposed in 3GPP standard [31] which consists of two kinds of relay: moving relay nodes [32], [33] and relay-users [34]. This concept provides great potential for $5 \mathrm{G}$ because it deals with the high implement cost of fixed relay. 
Full-duplex relaying communication in CRNs is introduced with the aim of inheriting the advantages of CNs and FD system having been intensively and widely studied in the literature [6], [12], [36]. Fig. 8 demonstrates the case of a FD relaying system in CRNs, where the secondary network is a FD relaying system, operating under underlay scheme. Due to the transmit power constraint of ST, in many cases the secondary receiver (SR) can not directly receive signal from the secondary transmitter (ST), so that a relay node is required. To enhance data rate and spectral efficiency, FD relay (FD-R) is considered instead of HD relay. Certainly, FD-R has to perform a self-interference suppression scheme to overcome the self-interference phenomenon at the relay.

Notably, the spectrum allocation of D2D communication is mostly managed by BS, whereas CRNs is fully autonomous by SUs. Thus, SUs are able to detect the usable spectrum, select the best one, and adjust parameters such as pre-coding and transmit power.

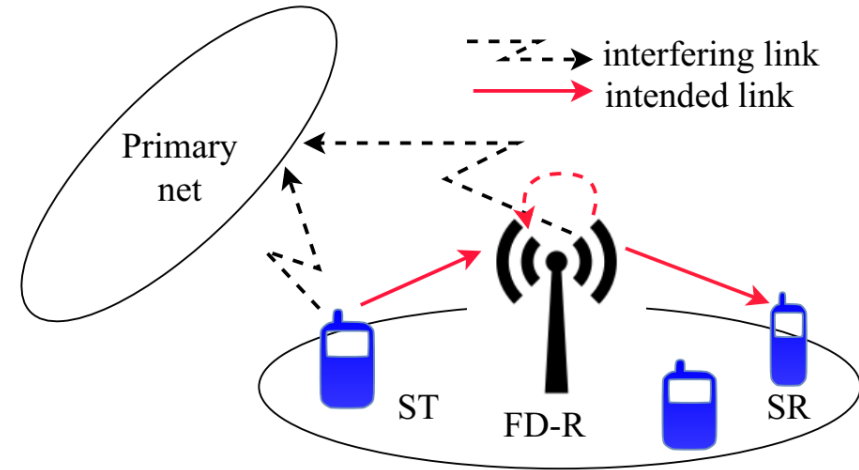

Secondary net

Figure 8. FDR in CRN system (ST: Secondary transmitter, FD-R : Ful-duplex relay, SR: Secondary receiver)

\section{Device-to-Device Communications}

Along with massive MIMO and CRNs, D2D communication has emerged as a promising solution for improving spectral efficiency of cellular networks (CelNs) [37]. It also provides a solution for decreasing overload of data traffic over CelNs and reducing transmission delay by directly communicating among devices [37]. Based on the kind of spectrum, D2D can be classified into two groups including in-band and outband D2D communication [8].

\subsection{Out-band D2D communications}

For out-band D2D communication, D2D devices (DUs) use unlicensed spectrum, such as Wi-fi, Bluetooth and ZigBee, for transmitting and receiving data. The benefit of this type is no interference between D2D and CelN. 
In terms of spectrum access policies, a device can occupy an unlicensed spectrum under the management of CelNs, called controlled out-band communication, or without any involvement of CelNs, called autonomous out-band communication [8]. For the former, the D2D communication performance can be controlled to improve the reliability and to ensure QoS. In contrast, autonomous out-band communication independently operates from CelN, which means that less overhead of CelNs, but the QoS cannot be controlled [38].

\subsection{In-band D2D communications}

Different from out-band, DUs share spectrum with cellular users (CelUs) in two modes: underlay mode (UMod) and overlay mode (OMod) [8]. The advantage of this paradigm is that CelNs are able to manage performance of D2D communications.

For OMod, the radio and time resources are allocated to DUs and CelUs such that there is no interference caused by D2D communication on CelNs and vice versa. As a result, interference management is not needed for this case, but the spectrum efficiency is not optimal and CelUs cannot exploit the full capacity of CelNs that can provide [8].

For UMod, both DUs and CelUs can use the same frequency and the same time slot for their transmissions, resulting in existing interference among them. Thus, the system needs to implement an additional scheme to partly or totally cancel the interference, making the system more complicated. With a sufficiently good interference management, the spectrum efficiency of this mode is improved significantly [38].

TABLE 2. Some key features of in-band and out-band D2D communications

\begin{tabular}{|l|c|c|c|c|}
\hline \multicolumn{1}{|c|}{ Advantages } & \multicolumn{2}{c|}{ In-band } & \multicolumn{2}{c|}{ Out-band } \\
\cline { 2 - 5 } & underlay & overlay & control & auto \\
\hline Enable to control the performance of DUs & $\mathrm{Y}$ & $\mathrm{Y}$ & $\mathrm{Y}$ & $\mathrm{N}$ \\
\hline Improve spectral efficiency & $\mathrm{Y}$ & $\mathrm{Y}$ & $\mathrm{N}$ & $\mathrm{N}$ \\
\hline CelNs' spectral efficiency is optimal & $\mathrm{Y}$ & $\mathrm{N}$ & $\mathrm{NA}$ & $\mathrm{NA}$ \\
\hline $\begin{array}{l}\text { Enable DUs and simultaneously operate on the same } \\
\text { spectrum of CelNs }\end{array}$ & $\mathrm{Y}$ & $\mathrm{N}$ & $\mathrm{NA}$ & $\mathrm{NA}$ \\
\hline
\end{tabular}

Along with EE, reducing energy consumption and prolonging battery life are also two important aspects in 5G visions [2]. Among many solutions, wireless power transfer (WPT) and energy harvesting (EH) have drawn great attention from the research community. The main concept of $\mathrm{EH}$ is to harvest and convert unused energy to useful energy to charge batteries. For natural energies such as solar and wind, they are free but strongly depend on weather and unable to exactly predict, meanwhile wireless communication systems require high reliability. For that reason, radio frequency (RF) 
signals, which are independent of natural conditions, become a potential resource [39],[40]. For WPT, energy is wirelessly transmitted to an intended receiver and it is usually fully controlled by transmitters.

Although EH and WPT have been considered as a promising technique, there still are some challenges that need to be solved to make it practical. For example, the amount of harvested energy is very small. Thus, the application of EH and WPT, recently, has been considered in D2D communications due to low-power devices and short transmission.

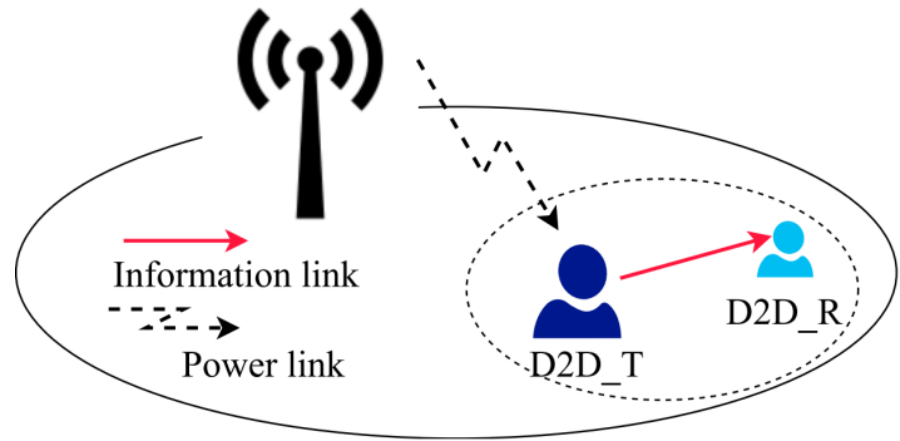

Figure 9. An example of EH based D2D communication system.

\section{Conclusions}

Throughout the paper, we have presented a comprehensive study on the emerging techniques for 5G including FDR in CRNs, D2D communications and CF massive MIMO. Some potential directions could be developed from the paper are listed as follows:

- Due to the randomness of location of users in CF massive MIMO, deploying stochastic geometry theory to model the system is an potential topic.

- Resource allocation for multigroup multicast CF massive MIMO to reduce the inter-user interference.

- In the near future, the need of directly communicating between devices is predicted to increase. Thus, considering D2D system in CF massive MIMO is also a promising direction.

- The development of low power devices makes energy harvesting become more practical. However, EHC in CF massive MIMO has not been studied in the literature. 
Toan Xuan Doan, Thanh Quoc Trinh - Volume 2 - Issue 4-2020, p. 348-362.

\section{References}

[1] D. Molkdar, W. Featherstone, S. Lambotharan (2002). An overview of EGPRS: the packet data component of EDGE. J. Electron. Commun. Eng., 14(1),21-38.

[2] GSMA Intelligence (2014). Understanding 5G: Perspectives on future technological advancements in mobile. White paper.

[3] M. Agiwal and A. Roy and N. Saxena (2016). Next Generation 5G Wireless Networks: A Comprehensive Survey. IEEE Commun. Surveys Tuts., 18(3), 1617-1655.

[4] F. Akyildiz and W. y. Lee and M. C. Vuran and S. Mohanty (2008). A survey on spectrum management in cognitive radio networks. IEEE Commun. Mag., 46(4), 40-48.

[5] B. Wang and K. J. R. Liu (2011). Advances in cognitive radio networks: A survey. IEEE J. Sel. Topics Signal Process, 5(1), 5-23.

[6] M. Amjad and F. Akhtar and M. H. Rehmani and M. Reisslein and T. Umer (2017). FullDuplex Communication in Cognitive Radio Networks: A Survey. IEEE Commun. Surveys Tuts, 19(4), 2158-2191.

[7] Z. Zhang and K. Long and A. V. Vasilakos and L. Hanzo (2016). Full-Duplex Wireless Communications: Challenges, Solutions, and Future Research Directions. Proc. of the IEEE, 104(7), 1369-1409.

[8] Asadi and Q. Wang and V. Mancuso (2014). A Survey on Device-toDevice Communication in Cellular Networks. IEEE Commun. Surveys Tuts., 16(4), 1801-1819.

[9] H. Q. Ngo and A. Ashikhmin and H. Yang and E. G. Larsson and T. L. Marzetta (2015). Cell-Free Massive MIMO: Uniformly great service for everyone. in Proc. Int. Workshop Signal Process. Adv. Wireless Commun., pp. 201-205.

[10] H. Q. Ngo and L. N. Tran and T. Q. Duong and M. Matthaiou and E. G. Larsson (2018). On the Total Energy Efficiency of Cell-Free Massive MIMO. IEEE Trans. Green Commun. Netw, 2(1), 25-39.

[11] H. Q. Ngo and A. Ashikhmin and H. Yang and E. G. Larsson and T. L. Marzetta (2017). Cell-Free Massive MIMO Versus Small Cells. IEEE Trans. Wireless Commun, 16(3), 1834-1850.

[12] H. Ju and E. Oh and D. Hong (2009). Improving efficiency of resource usage in two-hop full duplex relay systems based on resource sharing and interference cancellation. IEEE Trans. Wireless Commun., 8(8), 3933-3938.

[13] R. Lopez-Valcarce and E. Antonio-Rodriguez and C. Mosquera and F. Perez-Gonzalez (2012). An Adaptive Feedback Canceller for Full-Duplex Relays Based on Spectrum Shaping. IEEE J. Sel. Areas Commun, 30(8), 1566-1577.

[14] G. Interdonato and H. Q. Ngo and E. G. Larsson and P. Frenger (2016). How Much Do Downlink Pilots Improve Cell-Free Massive MIMO? IEEE Global Communications Conference (GLOBECOM), p.1-7.

[15] M. Bashar, K. Cumanan, A. G. Burr, H. Q. Ngo, and M. Debbah (2018). Cell-free Massive MIMO with limited backhaul, in Proc. IEEE ICC, pp. 1-7. 
[16] S. Shamai and B. M. Zaidel (2001). Enhancing the cellular downlink capacity via coprocessing at the transmitting end. in Proc. IEEE VTC-Spring, vol. 3, pp. 1745-1749.

[17] Osseiran, J. F. Monserrat, and P. Marsch (2016). 5G Mobile and Wireless Communications Technology. Cambridge University Press, ch. 9, Coordinated Multi-Point Transmission in $5 \mathrm{G}$.

[18] Shidong Zhou and Ming Zhao and Xibin Xu and Jing Wang and Yan Yao (2003). Distributed wireless communication system: a new architecture for future public wireless access. IEEE Commun. Mag., 41(3), 108-113.

[19] J. Mitola and G. Q. Maguire (1999). Cognitive radio: making software radios more personal. IEEE Pers. Commun., 6(4), 13-18.

[20] G. I. Tsiropoulos and O. A. Dobre and M. H. Ahmed and K. E. Baddour (2016). Radio Resource Allocation Techniques for Efficient Spectrum Access in Cognitive Radio Networks. IEEE Commun. Surv. Tutor, 18(1), 824-847.

[21] Goldsmith and S. A. Jafar and I. Maric and S. Srinivasa (2009). Breaking Spectrum Gridlock With Cognitive Radios: An Information Theoretic Perspective. Proc. IEEE, 97(5), 894-914.

[22] V. Valenta and R. Maršalek and G. Baudoin and M. Villegas ' and M. Suarez and F. Robert (2010). Survey on spectrum utilization in Europe: Measurements, analyses and observations. in Proc. 5th Int. Conf. CrownCom, Jun. 2010.

[23] S. Haykin (2005). Cognitive radio: brain-empowered wireless communications. IEEE J. Sel. Areas Commun., 23(2), 201-220.

[24] S. Srinivasa and S. A. Jafar (2007). Cognitive Radios for Dynamic Spectrum Access - The Throughput Potential of Cognitive Radio: A Theoretical Perspective. IEEE Commun. Mag., 45(5), 73-79.

[25] M. O. Hasna and M. S. Alouini (2003). End-to-end performance of transmission systems with relays over Rayleigh-fading channels. IEEE Trans. Wireless Commun., 2(6), 1126113.

[26] S. Ikki and M. H. Ahmed (2007). Performance Analysis of Cooperative Diversity Wireless Networks over Nakagami-m Fading Channel. IEEE Commun. Lett., 11(4),334-336.

[27] H. A. Suraweera and H. K. Garg and A. Nallanathan, "Performance Analysis of Two Hop Amplify-and-Forward Systems with Interference at the Relay," IEEE Commun. Lett., vol. 14, no. 8, pp. 692-694, Aug. 2010.

[28] J. H. Lee and O. S. Shin (2010). Full-duplex relay based on block diagonalisation in multiple-input multiple-output relay systems. IET Comm., 4(15), 1817-1826.

[29] J. N. Laneman and D. N. C. Tse and G. W. Wornell (2004). Cooperative diversity in wireless networks: Efficient protocols and outage behavior. IEEE Trans. Inf. Theory, 50(12), 3062-3080.

[30] T. Q. Duong and Vo Nguyen Quoc Bao and H. j. Zepernick (2009). On the performance of selection decode-and-forward relay networks over Nakagami-m fading channels. IEEE Commun. Lett., 13(3), 172-174. 
Toan Xuan Doan, Thanh Quoc Trinh - Volume 2 - Issue 4-2020, p. 348-362.

[31] S. Yang and X. Xu and D. Alanis and S. Xin Ng and L. Hanzo (2016). Is the LowComplexity Mobile-Relay-Aided FFR-DAS Capable of Outperforming the HighComplexity CoMP?. IEEE Trans. Veh. Technol., 65(4), 2154-2169.

[32] M. S. Pan and T. M. Lin and W. T. Chen (2015). An Enhanced Handover Scheme for Mobile Relays in LTE-A High-Speed Rail Networks. IEEE Trans. Veh. Technol., 64(2), 743-75.

[33] D. Hwang and D. I. Kim and S. K. Choi and T. J. Lee (2015). UE Relaying Cooperation Over D2D Uplink in Heterogeneous Cellular Networks. IEEE Trans. Commun., 63(12), 4784-4796.

[34] S. S. Nam and M. S. Alouini and S. Choi (2018). Iterative Relay Scheduling With Hybrid ARQ Under Multiple User Equipment (Type II) Relay Environments. IEEE Access, vol. 6, pp. 6455-6463.

[35] M. G. Khafagy, M. S. Alouini, and S. Ä1ssa (2018). Full-duplex relay selection in cognitive underlay networks. IEEE Trans. Commun., pp. 1-1.

[36] D. Feng and L. Lu and Y. Yuan-Wu and G. Y. Li and S. Li and G. Feng (2014). Device-todevice communications in cellular networks. IEEE Commun. Mag., 52(4), 49-5.

[37] F. Jameel and Z. Hamid and F. Jabeen and S. Zeadally and M. A.Javed (2018). A Survey of Device-to-Device Communications: Research Issues and Challenges. IEEE Commun. Surveys Tuts., pp. 1-1,.

[38] S. Sudevalayam and P. Kulkarni (2011). Energy Harvesting Sensor Nodes: Survey and Implications. IEEE Commun. Surveys Tuts., 13(3), 443-461.

[39] R. V. Prasad and S. Devasenapathy and V. S. Rao and J. Vazifehdan (2014). Reincarnation in the Ambiance: Devices and Networks with Energy Harvesting. IEEE Commun. Surveys Tuts., 16(1), 195-213. 